\title{
BMJ Open Longitudinal effect of nocturnal $R-R$ intervals changes on cardiovascular outcome in a community-based cohort
}

\author{
Abdulghani Sankari, ${ }^{1,2}$ Laurel Anne Ravelo, ${ }^{3}$ Scott Maresh, ${ }^{1}$ Nawar Aljundi, ${ }^{1}$ \\ Bander Alsabri, ${ }^{1}$ Serene Fawaz, ${ }^{1}$ Mulham Hamdon, ${ }^{1}$ Ghazwan Al-kubaisi, ${ }^{1}$ \\ Erika Hagen, ${ }^{3}$ M Safwan Badr, ${ }^{2}$ Paul Peppard ${ }^{3}$
}

To cite: Sankari A, Ravelo LA, Maresh S, et al. Longitudinal effect of nocturnal $R-R$ intervals changes on cardiovascular outcome in a communitybased cohort. BMJ Open 2019:9:e030559. doi:10.1136/ bmjopen-2019-030559

- Prepublication history and additional material for this paper are available online. To view please visit the journal (http:// dx.doi.org/10.1136/bmjopen2019-030559).

Received 20 March 2019 Revised 28 May 2019 Accepted 6 June 2019

Check for updates

(C) Author(s) (or their employer(s)) 2019. Re-use permitted under CC BY-NC. No commercial re-use. See rights and permissions. Published by BMJ.

${ }^{1}$ Internal Medicine, Wayne State University School of Medicine, Detroit, Michigan, USA ${ }^{2}$ Division of Pulmonary Critical Care and Sleep Medicine, Department of Internal Medicine, Wayne State University School of Medicine, Detroit, Michigan, USA

${ }^{3}$ Population Health Sciences, University of Wisconsin, Madison, Wisconsin, USA

Correspondence to Dr Abdulghani Sankari; asankari@wayne.edu

\section{ABSTRACT}

Rationale Sleep-disordered breathing (SDB) is strongly linked to adverse cardiovascular outcomes (cardiovascular diseases (CVD)). Whether heart rate changes measured by nocturnal R-R interval (RRI) dips (RRI dip index (RRDI)) adversely affect the CVD outcomes is unknown.

Objectives To test whether nocturnal RRDI predicts CVD incidence and mortality in the Wisconsin Sleep Cohort study (WSCS), independent of the known effects of SDB on beat-to-beat variability.

Methods The study analysed electrocardiograph obtained from polysomnography study to assess the nocturnal total RRDI (the number of RRI dips divided by the total recording time) and sleep RRDI (the number of RRI dips divided by total sleep time). A composite CVD risk as a function of total and sleep RRDI was estimated by Cox proportional hazards in the WSCS.

Results The study sample consisted of 569 participants from the WSCS with no prior CVD at baseline were followed up for up to 15 years. Nocturnal total RRDI (10unit change) was associated with composite CVD event(s) (HR, 1.24 per 10 -unit increment in RRDI $(95 \% \mathrm{Cl} 1.10$ to 1.39), $p<0.001)$. After adjusting for demographic factors (age $58 \pm 8$ years old; $53 \%$ male; and body mass index $31 \pm 7 \mathrm{~kg} / \mathrm{m}^{2}$ ), and apnoea-hypopnoea index (AHI 4\%), individuals with highest total nocturnal RRDI category $(\geq 28 \mathrm{vs}<15$ dips/hour) had a significant HR for increased incidence of CVD and mortality of $7.4(95 \% \mathrm{Cl} 1.97$ to $27.7), p=0.003)$. Sleep RRDI was significantly associated with new-onset CVD event(s) (HR, 1.21 per 10-unit increment in RRDI ( $95 \% \mathrm{Cl} 1.09$ to 1.35 ), $\mathrm{p}<0.001$ ) which remained significant after adjusting for demographic factors, AHI 4\%, hypoxemia and other comorbidities. Conclusion Increased nocturnal RRDI predicts cardiovascular mortality and morbidity, independent of the known effects of SDB on beat-to-beat variability. The frequency of RRDI is higher in men than in women, and is significantly associated with new-onset CVD event(s) in men but not in women.

\section{INTRODUCTION}

Sleep-disordered breathing (SDB) is a disorder characterised by the occurrence of recurrent episodes of apnoea and hypopnoea, resulting in a cascade of physiological responses including hypoxemia,

\section{Strengths and limitations of this study}

- It used a new method of detecting heart rate accelerations to assess its effect on the incidence of cardiovascular diseases (such as heart attack, heart failure, or need for cardiac procedures) and cardiovascular-related mortality.

- Using secondary analysis of a database of a prospective community cohort from the Wisconsin Sleep Cohort, we found that heart rate accelerations predict cardiovascular mortality and incidence of cardiovascular diseases.

- These results suggest that assessing the nocturnal ECG for heart accelerations may assist in predicting cardiovascular disease early on.

- The study was focused on individuals who had no prior pre-existing cardiac disease and were not on medications that affect heart rate such as Beta blocker; therefore, it may not be applicable to patients with current heart disease.

- This study lacks racial diversity as $95 \%$ was reported as a white race. Therefore, the results may not be generalisable to other races.

hypercapnia, intrathoracic pressure swings due to the inspiratory effort, activation of the sympathetic nervous system and arousal from sleep. ${ }^{1}$ In clinical practice, SDB is defined by the measurement of apnoea-hypopnoea index (AHI), as the average number of respiratory events divided by the total sleep time. Although AHI is easy to use, this measure discounts other physiological consequences of the respiratory events that may be important, including associated hypoxemia and arousals from sleep, as well as the cardiac autonomic disturbances throughout the night. ${ }^{2}$ Indeed, recent evidence showed that sub-type of excessively sleepy patients with moderate to severe SDB have significantly increased the risk for prevalent and incident cardiovascular events indicating the central role of sleep disruption in increased CVD risk. ${ }^{3}$ In addition, a recent study found that 
varying hypopnoea definitions in the general population can affect the risk stratification of cardiovascular disease in patients with SDB. ${ }^{45}$

While recent reports included measurements of sleep fragmentation and respiratory event duration as a surrogate of arousal threshold ${ }^{6}$ it did not include direct measurements of sympathetic activity and heart rate changes related to these events and its physiological stressors. Nocturnal heart rate variability, not day-time, is a heritable phenotype ${ }^{7}$ independent of covariates, suggesting that genetic factors play an important role in controlling these cardiovascular risk factors. ${ }^{8}$ Therefore, R-R interval (RRI), a time domain measure of heart rate variability, may reflect a physiological trait that predicts the risk of adverse cardiovascular outcomes, otherwise missed by SDB severity classification using traditional AHI and desaturation criteria. ${ }^{9}$ However, the long-term effect of heart rate changes during sleep on the cardiovascular outcome and mortality is unknown.

The objectives of this study were to examine whether RRI or heart rate accelerations can serve as predictors of cardiovascular disease in the Wisconsin Sleep Cohort study (WSCS), a prospective community cohort. We hypothesised that increased nocturnal RRI dip index (RRDI) would be associated with increased cardiovascular disease (CVD) or mortality independent of the known effects of SDB on beat-to-beat variability. Results of this study have been previously reported in the form of an abstract. ${ }^{1011}$

\section{METHODS}

\section{Participants}

We studied individuals from the WSCS. The protocols of the WSCS were approved by the Health Sciences Institutional Review Board of the University of Wisconsin-Madison. All participants provided written informed consent. Sampling and data collection protocols of the WSCS have been described previously. ${ }^{12}$

\section{Cohort description}

The WSCS comprises 1546 adult employees of state agencies, ages 30-60 years old at the Cohort's inception, which underwent attended in-laboratory overnight polysomnography (PSG) and provided health-related questionnaires approximately every 4 years. Data presented here were collected from August of 2000 through August 2016 (the period when digital PSG recording systems were in use by the WSCS). The most recent available PSG study was used for analysis. WSCS participants were eligible to be included in the study if they had full PSG with adequate ECG recording, not treated for SDB, had no prior CVD event and did not use beta blockers or chronotropic drugs (online supplementary table 1S) on the night of the sleep study or at any other point during follow-up.

\section{Patient and public involvement}

This study was a secondary analysis for pre-existing data from an established cohort of the Wisconsin study. Therefore, participants were not involved in the design, recruitment, or conduct of this study.

\section{Predictor}

The main predictor variable is the hourly rate of RRI changes assessed over an entire night's sleep period. The recorded ECG signals were retrieved from PSG to measure the RRI, which are time intervals between successive pairs of QRS complexes, by using software for the detection of $\mathrm{R}$ waves in LabChart 7 with heart rate variability Module (AD Instruments, Colorado Springs, Colorado, USA) (figure 1). In this program, ECG signal was examined and retrieved to a MatLab R2017a program (MathWorks, Natick, Massachusetts, USA) developed and validated by our group to obtain RRI signal for the entire night (figure 2). ${ }^{13} 14$ The RRI dips, defined by a decreased RRI compared with the average RRI for the corresponding $1 \mathrm{~min}$ segment as a baseline, were collected. Given that $90 \%$ dips threshold correlated previously with most respiratory events (apneic and non-apneic respiratory events, defined below), ${ }^{14}$ total RRI dips index (RRDI) was defined by the number of RRI dips below the $90 \%$ baseline divided by the total PSG recording time in hours (from light on to light out), regardless of wake or sleep stages. Sleep RRDI for non-rapid eye movement (REM) and REM stages combined were defined by the number of RRI dips below the $90 \%$ baseline divided by the total sleep time in hours (for both REM and non-REM sleep stages). Subsequently, sleep RRDI was calculated for specific sleep stages for REM and non-REM, respectively. In subgroup analysis, the gender differences in total and sleep RRDI were compared between men and women.

The person performing the analysis was blinded to the participant's demographic information. The RRDI values were examined as a continuous variable and as a categorical variable divided into tertiles (lower $25 \%$ (low), middle $50 \%$ (medium) and upper $25 \%$ (high)).

\section{Outcome}

Incident CVD events were death related to CVD, self-reported physician-diagnosed heart attack, heart failure, or a CVD procedure (angioplasty, stent, pacemaker, bypass, or defibrillation). Information on CVD events, including the date of the event, was obtained on the overnight health questionnaire. Death certificates and cause of mortality in the cohort were obtained by matching social security numbers with two data sources: The National Death Index and the Wisconsin State Bureau of Health Information and Policy, Phial Records Section. All included participants in this study were able to complete the follow-up information. The censored analysis was used, and the data is censored at the last visit. If multiple events were reported over the 

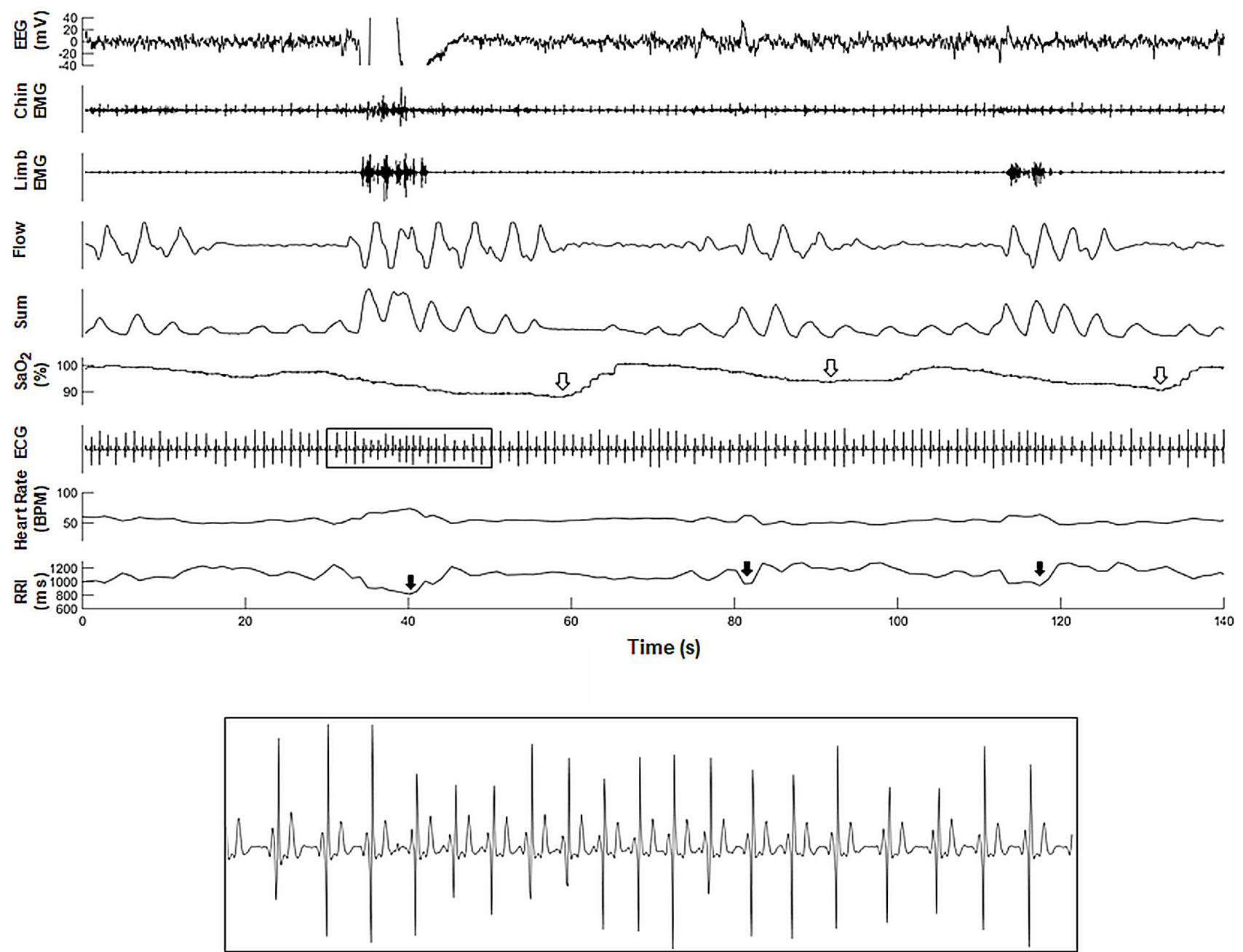

Figure 1 (A) A representative polygraph from one subject in the WSCS that illustrate changes in respective heart rate and computed RRI tracing during sleep. (B) A magnified segment of ECG illustrating RRI following respiratory event (apnoea). The $\mathrm{RRI}$ tracing was retro-graphed from the exported signal that calculates RRI from raw ECG recordings. Open arrows indicate oxygen desaturation following apnoea and closed arrows indicate RRI dip following apnoea. EEG, electroencephalogram; EMG, electromyogram;; RRI, R-R interval; WSCS, Wisconsin Sleep Cohort Study.

course of follow-up, the first reported event was used in this analysis.

\section{Covariates}

Participants underwent a baseline overnight 18-channel PSG (Grass model 78; Quincy, Massachussets, USA) at the University of Wisconsin-Madison Clinical Research Unit using a standard protocol. ${ }^{15}$ The PSG recorded sleep state using electroencephalography, electrooculography and electromyography; and breathing, using respiratory inductance plethysmography (Respitrace; Ambulatory Monitoring, Ardsley, New York, USA), nasal and oral airflow (ProTec thermocouples; Hendersonville, Tennessee, USA) and oxyhaemoglobin saturation, using pulse oximetry (Ohmeda Biox 3740, Englewood, Colorado, USA). Each $30 \mathrm{~s}$ epoch of the polysomnographic recordings was scored for sleep stage and apnoea and hypopnoea events by trained technicians and reviewed using standard criteria. ${ }^{15}$ Apnoea was defined as the cessation of nasal and oral airflow for $\geq 10 \mathrm{~s}$ and hypopnoea as a discernible reduction in breathing (sum of the chest and abdominal excursions) with a decrease in oxyhaemoglobin saturation of $\geq 4 \%$. The apnoea-hypopnoea index was calculated as the mean number of apnoea and hypopnoea events per hour of sleep.

\section{Statistical analysis}

Cox proportional hazards regression was used to estimate adjusted HRs and 95\% CIs for the association between RRDI and subsequent risk of an incident CVD event. ${ }^{16}$ Because of the strong dependence of CVD risk on age, Cox-regression models were based on age as the time scale, (the age when RRDI was measured and age at the event) allowing for left truncation (late entry). ${ }^{17}$ In addition to adjusting for age using this methodology, models 2 were adjusted for body mass index (BMI) and gender. Models 3 were adjusted for AHI $4 \%$ (as continuous and categorical variables (AHI $<5,5-15$, or $>15$ events / hour)). Subsequently, models 4 were adjusted for other factors: diabetes, hypertension, stroke, smoking, average 

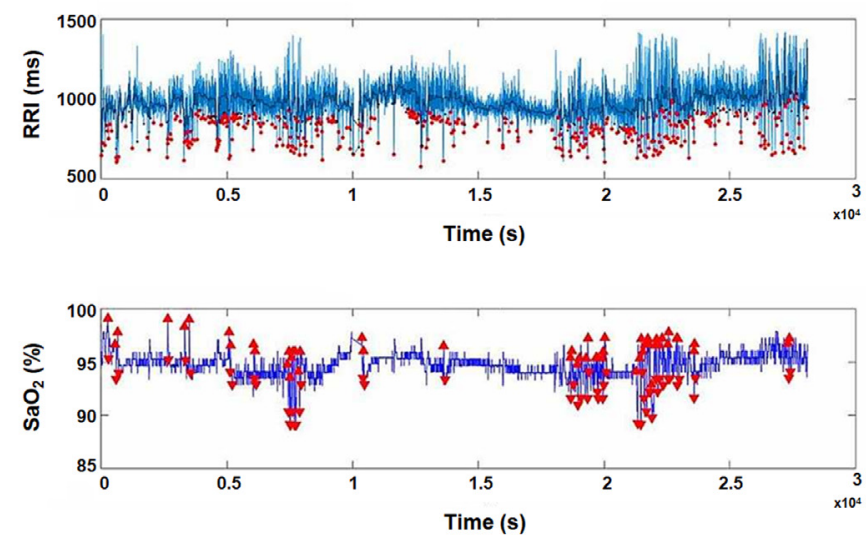

Figure 2 A representative computed data of RRI and oxygen saturation $\left(\mathrm{SaO}_{2}\right)$ from one individual during sleep. The red dots represent the RRI dips throughout the duration of the PSG recording (approximately 8 hours). The RRDI at $90 \%$ threshold for this participant was 54.5 dips/hour, the average heart rate was $61.1 \mathrm{bpm}$ and the ODI (3\%) was 2.3 de-saturations/hour (from the original PSG recording). The RRI tracing was retro-graphed from the exported signal that calculates $\mathrm{RRI}$ and $\mathrm{SaO}_{2}$ from raw ECG and pulse oximetry recordings, respectively. ODI, oxygen desaturation index; PSG, polysomnography; RRI, R-R intervals; RRDI, RRI dips index; $\mathrm{SaO}_{2}$, oxygen saturation.

HR, \% TST $<90 \%$, Kaplan-Meier techniques were used to compare survival across RRDI categories. ${ }^{18}$

\section{RESULTS}

\section{Baseline characteristics}

Table 1 presents the baseline characteristics of the eligible participants. A total of 1440 sleep studies were examined for inclusion in this study as depicted in figure 3 . The final sample included 569 participants (one sleep study per participant) after excluding those on continuous positive airway pressure (CPAP) treatment, individuals who had a prior history of cardiovascular disease, use of beta blocker the night of the study or in other visits during the study, lack of follow-up or if they had events before PSG. RRDI (at 90\% threshold) significantly correlated with the following sleep parameters: AHI $4 \%$, periodic legs movements index (PLMI) and respiratory electroencephalogram arousals $(\mathrm{p}<0.001)$ (table 2$)$.

\section{CVD incidence-association with total RRDI}

Using Cox Proportional Hazards Model, continuous total RRDI (with 90\% threshold for RRI dips) was significantly associated with new-onset CVD event(s) (HR, 1.24 per 10-unit increment in RRDI (95\% CI 1.10 to 1.39$), \mathrm{p}<0.001$ ) which remained significant after adjustment for age, BMI and gender (model 1) and the addition of AHI 4\% (model 3) (as depicted in table 3). Lower thresholds (80\%, $70 \%$ and $60 \%$ ) of RRDI correlated with total RRDI $90 \%$ but were less sensitive in predicting CVD (less than five individuals attained RRDI $>20$ dips/hour). The association between total RRDI at $90 \%$ threshold and the incidence of new-onset
Table 1 Baseline characteristics

\begin{tabular}{|c|c|}
\hline Characteristics & Value \\
\hline$n$ & 569 \\
\hline Age in years, mean (SD) range & 58 (8) 39-79 \\
\hline $\begin{array}{l}\text { Body mass index in } \mathrm{kg} / \mathrm{m}^{2} \text {, mean (SD) } \\
\text { range }\end{array}$ & $31(7) 18-66$ \\
\hline Years to event/censor, mean (SD) range & $8(4) 0.1-15$ \\
\hline Males, n (\%) & $300(53)$ \\
\hline \multicolumn{2}{|l|}{ Apnoea-hypopnoea Index, n (\%) } \\
\hline$<5$ & $253(44)$ \\
\hline $5-15$ & $168(30)$ \\
\hline$>15$ & $148(26)$ \\
\hline Diabetes, n (\%) & $32(6)$ \\
\hline Hypertension, n (\%) & $191(34)$ \\
\hline Stroke, n (\%) & $11(2)$ \\
\hline $\begin{array}{l}\text { Antihypertensive medication use (excluding } \\
\text { Beta Blockers or any chronotropic } \\
\text { medication), } \mathrm{n}(\%)\end{array}$ & $133(24)$ \\
\hline \multicolumn{2}{|l|}{ Smoking, n (\%) } \\
\hline Current & $63(11)$ \\
\hline Past & $213(37)$ \\
\hline Never & $293(52)$ \\
\hline White race, $\mathrm{n}(\%)$ & $538(97)$ \\
\hline $\begin{array}{l}\text { Number of alcoholic drinks per week, } \\
\text { mean (SD) range }\end{array}$ & 4 (5) 0-32 \\
\hline Total sleep time, minutes, mean (SD) range & $368(61) 30-514$ \\
\hline Per cent stage 1 sleep, mean (SD) & $10.6(6.5)$ \\
\hline Per cent stage 2 sleep, mean (SD) & $65.0(9.3)$ \\
\hline Per cent stage 3,4 sleep, mean (SD) & $7.8(8.0)$ \\
\hline Per cent REM sleep, mean (SD) & $16.6(6.4)$ \\
\hline Mean $\mathrm{SaO}_{2}$, mean (SD) & $95.4(1.8)$ \\
\hline Mean desaturation, mean (SD) & $4.5(1.5)$ \\
\hline $\begin{array}{l}\text { Percentage of total sleep time below } 90 \% \\
\text { saturation, mean (SD) }\end{array}$ & $2.7(11.2)$ \\
\hline
\end{tabular}

REM, rapid eye movement; $\mathrm{SaO}_{2}$, oxygen saturation.

CVD remained significant after the adjustment for AHI 4\% (model 3) $(\mathrm{p}<0.001)$. Total RRDI category 3 ( $\geq 28.4$ /hour vs $<15.1 \mathrm{dips} /$ hour) was associated with increased CVD hazards risk of 6.1 (95\% CI 1.7 to 27.7, $\mathrm{p}=0.005)$ and remained significant after adjustment for AHI 4\% ( $\mathrm{p}=0.003)$. Continuous total RRDI 90\% remained significant (HR, 1.22 (per 10-unit increment in RRDI (95\% CI 1.08, 1.37), $\mathrm{p}=0.001)$ after additionally adjusted for diabetes, hypertension, stroke, smoking, average heart rate and $\%$ total time with oxygen saturation less than 90\% (model 4) (as depicted in table 3).

Figure 4 illustrates the changes in CVD incidence and hazard ratios for total RRDI less than 15.1 dips per hour (as reference), RRDI 15.1-28.4 dips/hour (second tertile) and for the third group (tertile) of individuals with RRDI equal 
Excluded:

141 used Beta Blockers during PSG

86 used CPAP on the night of the study

\section{Excluded: \\ 68 had no follow up \\ 1 with bad ECG \\ 47 had an event before PSG}

\section{WSCS Studies were scored}

$\mathrm{N}=1440$

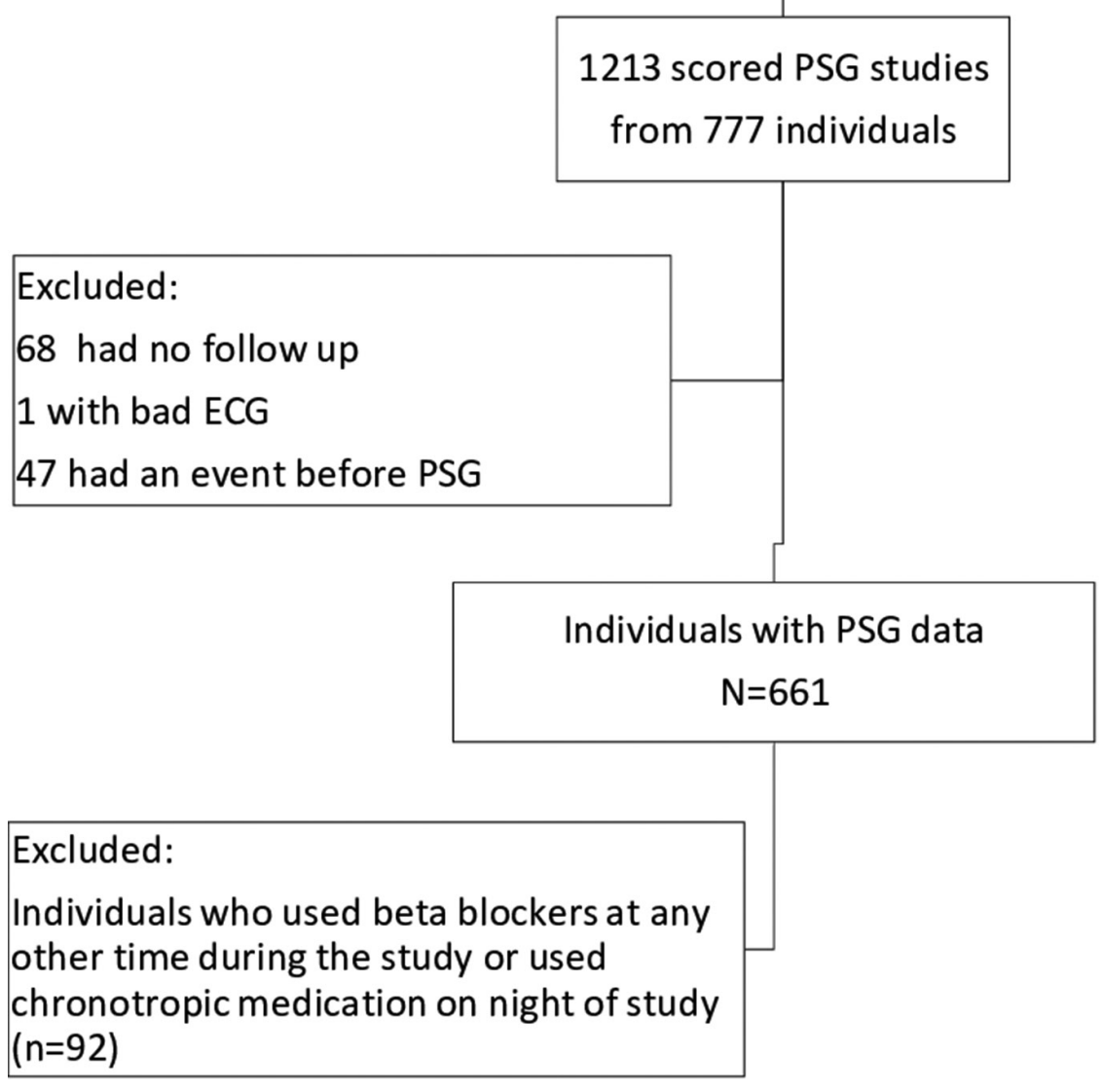

$\begin{gathered}\text { Individuals with PSG data included in } \\ \text { the anlaysis } \\ N=569\end{gathered}$

Figure 3 The WSCS study sample. CPAP, continuous positive airway pressure; PSG, polysomnography; WSCS, Wisconsin Sleep Cohort Study.

or more than 28.4 dips/hour. Kaplan-Meier survival curves (figure 5) illustrate decreased CVD event-free survival with increasing total RRDI category from RRDI less than 15.1 to RRDI equal or more than 28.4 dips/hour.

CVD incidence-association with sleep RRDI

Using Cox Proportional Hazards Model, continuous sleep RRDI $90 \%$ was significantly associated with new-onset CVD event(s) (HR, 1.21 per 10-unit increment in RRDI (95\% CI 1.09 to 1.35$), \mathrm{p}<0.001)$ which remained significant after the model adjusted for age, BMI and gender (model 2) and the addition of AHI 4\% (model 3 ) (as depicted in table 4). RRDI category 3 ( $\geq 23.5 /$ hour vs $<9.0$ /hour) was associated with increased CVD hazards risk of 3.39 (95\% CI 1.06 to $10.85, \mathrm{p}=0.04$ ) and remained significant after adjustment for demographics and AHI 4\% (model 4) ( $\mathrm{p}=0.037)$. The relationship between sleep RRDI categories and CVD events were predominantly in non-REM sleep as depicted in table 5 . 
Table 2 Pearson correlation for RRDI (at 90\% threshold)

\begin{tabular}{lcc}
\hline & $\begin{array}{l}\text { Correlation } \\
\text { coefficients }\end{array}$ & P value \\
\hline AHI 4\% & 0.18 & $<0.0001$ \\
PLMI & 0.19 & $<0.0001$ \\
Respiratory arousal index & 0.24 & $<0.0001$ \\
Leg movement arousal index & 0.23 & $<0.0001$ \\
Spontaneous arousal index & -0.09 & 0.127 \\
\hline
\end{tabular}

AHI 4\%, apnoea-hypopnoea index based on hypopnoea associated with $4 \%$ desaturation; PLMI, periodic legs movement index; RRDI, R-R interval dips index.

Continuous RRDI 90\% during REM sleep was significant (HR, 1.19 per 10-unit increment in RRDI (95\% CI 1.07 to 1.32), $\mathrm{p}=0.001$ ) in the unadjusted model (model 1 ) and remained significant after the model adjusted for age, BMI and gender (model 2) and the addition of AHI $4 \%$ (model 3) (as depicted in table 6). However, sleep RRDI category 3 ( $\geq 24 /$ hour vs $<9.0 /$ hour) was only significant in the unadjusted model (model 1) with hazards risk of new CVD events of $2.92(\mathrm{p}=0.04)$.

Cardiovascular events were detected in 25 participants (4\%), of the sample, over a follow-up interval of 15 years with mean age 59 years old (range 41-80). Cardiovascular events consisted of heart failure, heart attack, CVD procedure (before any events), or CVD death (table 7). No difference between mean age at the start for those who had CVD versus not $(\mathrm{p}=0.58)$.

Gender effect on the association of CVD incidence with total and sleep RRDI

Table 8 presents the baseline characteristics of men and women participants and associated total and sleep RRDI.

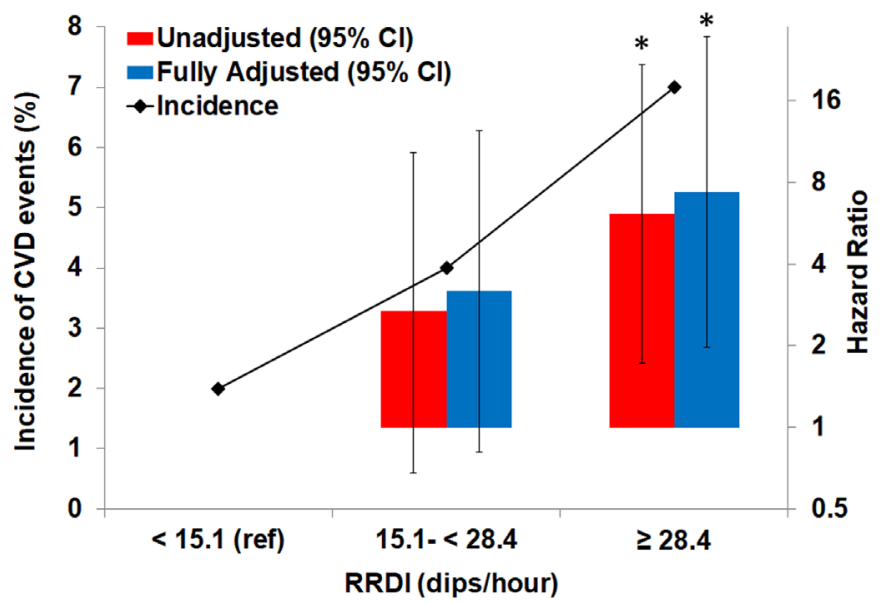

Figure 4 Incidence of composite CVD and hazard ratio across different total RRDI severity: Category 1 as a reference (RRDI <15.1), category 2 (RRDI 15.1-<28.4) and category 3 (RRDI $\geq 28.4)(n=569)$. ( $\left.{ }^{*}\right)$ versus unadjusted model (1) RRDI $<15.1$ dips/hour, $\mathrm{p}<0.01 ;\left(^{\star \star}\right)$ versus adjusted model (3) RRDI $<15.1$ dips/hour, $\mathrm{p}<0.01$. CVD, Cardiovascular disease ; RRDI, R-R interval dips index.

While BMI was higher in women (32.0 \pm 7.0 vs. $30.0 \pm 5.0$ $\left.\mathrm{kg} / \mathrm{m}^{2}, \mathrm{p}=0.0001\right)$, men had higher AHI, total RRDI and sleep RRDI than women $(p<0.01)$. Using Cox Proportional Hazards adjusted model (3) (for age, body mass index and AHI 4\%), continuous total RRDI 90\% was significantly associated with new-onset CVD event(s) in men (HR, 1.22 per 10-unit increment in RRDI (95\% CI 1.06 to 1.40 ), $\mathrm{p}<0.001$ ) but not in women (as depicted in Table 8). Likewise, continuous sleep RRDI $90 \%$ was significantly associated with new-onset CVD event(s) in men (HR, 1.19 per 10-unit increment in RRDI (95\% CI 1.04 to 1.36 ), $\mathrm{p}<0.05$ ) but not

Table 3 The adjusted time to event Cox Proportional Hazards Models for total RRDI predicting the incidence of CVD event

$\begin{array}{ll}\text { CVD } & \text { HR }(95 \% \mathrm{Cl}) \\ \text { events } & \mathrm{p} \text { value }\end{array}$

\begin{tabular}{|c|c|c|c|c|c|}
\hline & n (\%) & $\begin{array}{l}\text { Unadjusted model } \\
\text { (1) }\end{array}$ & Adjusted model (2) & Adjusted model (3) & Adjusted model (4) \\
\hline $\begin{array}{l}\text { Continuous RRDI (10- } \\
\text { unit increment) }\end{array}$ & $\begin{array}{l}24 / 569 \\
(4)\end{array}$ & $\begin{array}{l}1.24(1.10 \text { to } 1.39) \\
0.0003\end{array}$ & $\begin{array}{l}1.22(1.08 \text { to } 1.38) \\
0.0018\end{array}$ & $\begin{array}{l}1.23(1.11 \text { to } 1.38) \\
0.0007\end{array}$ & $\begin{array}{l}1.22(1.08 \text { to } 1.37) \\
0.0012\end{array}$ \\
\hline \multicolumn{6}{|l|}{ RRDI Category } \\
\hline $\begin{array}{l}\text { Tertile } 1 \\
(<15.1)\end{array}$ & $\begin{array}{l}3 / 187 \\
(2)\end{array}$ & REF & REF & REF & REF \\
\hline $\begin{array}{l}\text { Tertile } 2 \\
(15.1-<28.4)\end{array}$ & $\begin{array}{l}7 / 194 \\
(4)\end{array}$ & $\begin{array}{l}2.66(0.68 \text { to } 10.34) \\
0.1586\end{array}$ & $\begin{array}{l}2.72(0.70 \text { to } 10.59) \\
0.1481\end{array}$ & $\begin{array}{l}3.16(0.81 \text { to } 12.40) \\
0.099\end{array}$ & $\begin{array}{l}3.22(0.80 \text { to } 12.93) \\
0.10\end{array}$ \\
\hline $\begin{array}{l}\text { Tertile } 3 \\
(\geq 28.4)\end{array}$ & $\begin{array}{l}14 / 188 \\
(7)\end{array}$ & $\begin{array}{l}6.11(1.72 \text { to } 21.72) \\
0.0052\end{array}$ & $\begin{array}{l}5.87 \text { (1.60 to } 21.46) \\
0.0075\end{array}$ & $\begin{array}{l}7.40 \text { (1.97 to } 27.73) \\
0.003\end{array}$ & $\begin{array}{l}8.99(2.35 \text { to } 34.40) \\
0.001\end{array}$ \\
\hline P-trend & & 0.0024 & 0.0045 & 0.0017 & 0.0006 \\
\hline
\end{tabular}

Model (1) is unadjusted. Model (2) is adjusted for age, sex and body mass index. Model (3) is additionally adjusted for age, sex, body mass index and $\mathrm{AHI}$ (4\% criteria). Model (4) is additionally adjusted for diabetes, HTN, stroke and smoking, average HR and \%TST<90\%. $\mathrm{AHI} 4 \%$, apnoea-hypopnoea index with hypopnoea scored if associated with at least 4\% desaturation (events/hour); CVD, cardiovascular disease; HTN, hypertension; RRDI, R-R interval dips index (dips/hour); \%TST<90, total sleep time spent less than $90 \%$ on oxygen saturation signal (\%). 


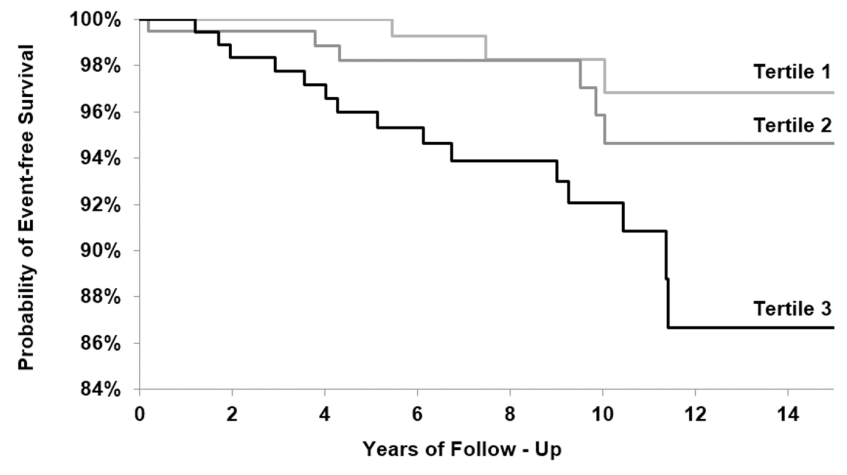

Log-Rank $p=0.0139$ for total RRDI

\begin{tabular}{|l|c|c|c|c|c|c|c|c|}
\hline Not at Risk & 0 & 2 & 4 & 6 & 8 & 10 & 12 & 14 \\
\hline Tertile 1 & 188 & 176 & 156 & 128 & 88 & 68 & 29 & 1 \\
\hline Tertile 2 & 193 & 182 & 160 & 134 & 106 & 79 & 35 & 2 \\
\hline Tertile 3 & 188 & 175 & 163 & 138 & 116 & 86 & 29 & 1 \\
\hline
\end{tabular}

Figure 5 Kaplan-Meier estimates of the likelihood of survival according to total RRDI severity: Category 1 as a reference (RRDI <15.1), category 2 (RRDI 15.1-<28.4) and category 3 (RRDI $\geq 28.4)$ ( $n=569)$; log-rank test for differences in survival by RRDI category; survival was lower for category 3 compared with group 1 and 2. RRDI is a mean number of RRI dips/hour of total recording time of PSG. PSG, polysomnography; RRDI, R-R interval dips index.

in women. Using total RRDI threshold of $20 \mathrm{dips} /$ hour or more from the adjusted model (model 3) was associated with increased CVD hazards risk of 4.34 in men only $(95 \%$ CI 1.32 to $14.34, \mathrm{p}=0.016$ ).

\section{DISCUSSION}

Our study prospectively examined the relationship of nocturnal beat-to-beat RRI changes, that is, brief accelerations in the heart rate, with long-term cardiovascular outcomes. The study revealed several important and novel findings. First, increased frequency of heart rate accelerations (RRDI) during sleep study was associated with the development of cardiac events or mortality in a prospective large community-based cohort of individuals over a follow-up interval of 15 years, who had no known heart disease at the time of their sleep study. Second, the relationship between total RRDI and incidence of CVD remained significant after adjusting for demographics, SDB severity using AHI 4\%, hypoxemia and other comorbidities. Third, the frequency of total RRDI was higher in men than in women and associated with CVD predominantly in men.

To our knowledge, this is the first community-based cohort that has shown an association between adverse cardiovascular outcomes and heart rate changes during sleep. Specifically, we found that the association between RRDI and incidence of new CVD events was independent of AHI (with a $4 \%$ desaturation threshold for hypopnoea scoring) and hypoxia. Prior studies assessed the relationship between AHI using different thresholds and adverse cardiovascular consequences. For example, in the Sleep Heart Health Study, Punjabi, et al. found an association of cardiovascular morbidity with SDB characterised by breathing events defined as having $\geq 4 \%$ desaturations, but not by SDB characterised by desaturations of less than $4 \% .{ }^{19}$ More recently, it has been found that the desaturation hypoxic burden related to respiratory events, measured by the integration of the severity of the desaturation and its length, predicted CVD mortality. ${ }^{20}$ Another study that examined heart rate variability during sleep found that SDB patients had shorter RRI and increased sympathetic burst frequency ( $49 \pm 4$ bursts $/ \mathrm{min}$ ) compared with control subjects. ${ }^{21}$ The authors speculated that abnormalities in heart rate and blood pressure variability might be implicated in the subsequent development of

Table 4 The adjusted time to event Cox Proportional Hazards Models for sleep RRDI predicting the incidence of CVD event

$\begin{array}{ll}\text { CVD } & \text { HR }(95 \% \mathrm{Cl}) \\ \text { events } & \text { p value }\end{array}$

n (\%) Unadjusted model (1) Adjusted model (2) Adjusted model (3) Adjusted model (4)

\begin{tabular}{llllll}
\hline Continuous RRDI & $24 / 569$ & $1.21(1.09$ to 1.35$)$ & $1.29(1.06$ to 1.33$)$ & $1.20(1.07$ to 1.34$)$ & $1.19(1.06$ to 1.33$)$ \\
(10-unit increment) & $(4)$ & 0.0006 & 0.0037 & 0.0015 & 0.003
\end{tabular}

RRDI category

\begin{tabular}{|c|c|c|c|c|c|}
\hline $\begin{array}{l}\text { Tertile } 1 \\
(<9.0)\end{array}$ & $\begin{array}{l}4 / 187 \\
(2)\end{array}$ & REF & REF & REF & REF \\
\hline $\begin{array}{l}\text { Tertile } 2 \\
(9.0-<23.5)\end{array}$ & $\begin{array}{l}9 / 194 \\
(5)\end{array}$ & $\begin{array}{l}2.61(0.79 \text { to } 8.57) \\
0.1144\end{array}$ & $\begin{array}{l}2.46(0.75 \text { to } 8.11) \\
0.1383\end{array}$ & $\begin{array}{l}2.66(0.80 \text { to } 8.77) \\
0.1092\end{array}$ & $\begin{array}{l}2.79(0.83 \text { to } 9.36) \\
0.10\end{array}$ \\
\hline $\begin{array}{l}\text { Tertile } 3 \\
(\geq 23.5)\end{array}$ & $\begin{array}{l}11 / 188 \\
(6)\end{array}$ & $\begin{array}{l}3.39(1.06 \text { to } 10.84) \\
0.0398\end{array}$ & $\begin{array}{l}2.94(0.91 \text { to } 9.56) \\
0.0729\end{array}$ & $\begin{array}{l}3.61(1.08 \text { to } 12.10) \\
0.0373\end{array}$ & $\begin{array}{l}4.00(1.17 \text { to } 13.68) \\
0.027\end{array}$ \\
\hline P-trend & & 0.0392 & 0.0768 & 0.0365 & 0.026 \\
\hline
\end{tabular}

Model (1) is unadjusted. Model (2) is adjusted for age, sex and body mass index. Model (3) is additionally adjusted for age, sex, body mass index and $\mathrm{AHI}(4 \%$ criteria). Model (4) is additionally adjusted for diabetes, HTN, stroke, and smoking, average HR, and \%TST<90\%. AHI 4\%, apnoea-hypopnoea index with hypopnoea scored if associated with at least 4\% desaturation (events/hour); CVD, cardiovascular disease; HTN, hypertension; RRDI, R-R interval dips index (dips/hour); \%TST<90, total sleep time spent less than $90 \%$ on oxygen saturation signal (\%). . 
Table 5 The adjusted time to event Cox Proportional Hazards Models for RRDI during non-REM sleep predicting the incidence of CVD event

\begin{tabular}{|c|c|c|c|c|}
\hline & $\begin{array}{l}\text { CVD } \\
\text { events }\end{array}$ & $\begin{array}{l}\text { HR }(95 \% \mathrm{Cl}) \\
\text { p value }\end{array}$ & & \\
\hline & n (\%) & Unadjusted model (1) & Adjusted model (2) & Adjusted model (3) \\
\hline \multicolumn{5}{|l|}{ RRDI category } \\
\hline $\begin{array}{l}\text { Tertile } 1 \\
(<8.5)\end{array}$ & $\begin{array}{l}4 / 187 \\
(2)\end{array}$ & REF & REF & REF \\
\hline $\begin{array}{l}\text { Tertile } 2 \\
(8.5-<22.6)\end{array}$ & $\begin{array}{l}9 / 194 \\
(5)\end{array}$ & $\begin{array}{l}2.69(0.82 \text { to } 8.86) \\
0.1040\end{array}$ & $\begin{array}{l}2.69(0.82 \text { to } 8.82) \\
0.1023\end{array}$ & $\begin{array}{l}2.85(0.87 \text { to } 9.36) \\
0.0849\end{array}$ \\
\hline $\begin{array}{l}\text { Tertile } 3 \\
(\geq 22.6)\end{array}$ & $\begin{array}{l}11 / 188 \\
(6)\end{array}$ & $\begin{array}{l}3.40(1.06 \text { to } 10.94) \\
0.0389\end{array}$ & $\begin{array}{l}3.11(0.96 \text { to } 10.06) \\
0.0577\end{array}$ & $\begin{array}{l}3.92(1.18 \text { to } 13.09) \\
0.0263\end{array}$ \\
\hline P-trend & & 0.0390 & 0.0612 & 0.0249 \\
\hline
\end{tabular}

Model (1) is unadjusted. Model (2) is adjusted for age, sex and body mass index. Model (3) is additionally adjusted for age, sex, body mass index and $\mathrm{AHI}(4 \%$ criteria).

AHI 4\%, apnoea-hypopnoea index with hypopnoea scored if associated with at least 4\% desaturation (events/hour); CVD, cardiovascular disease; REM, rapid eye movement; RRDI, R-R interval dips index (dips/hour).

cardiovascular disease in patients with SDB. The present study confirmed this association between the frequency of heart rate accelerations (RRDI) and adverse cardiovascular consequence in a prospective large cohort of individuals who had no known heart disease at the time of their sleep study.

The mechanism of increased incidence of CVD and association with RRDI can be explained by an increased sympathetic tone and autonomic arousals. First, sleep disturbances like SDB, periodic limb movements, insufficient sleep are all associated with an increased risk of CVD. These sleep disorders are commonly associated with impaired autonomic nervous system leading to increased sympathetic tone. ${ }^{22}$ Furthermore, sleep fragmentation due to autonomic or respiratory arousals from sleep increases the cardiac sympathetic tone activity resulting in a sudden elevation in vascular tone and heart rate generating a rise in arterial blood pressure. ${ }^{22}{ }^{23}$ The increased sympathetic tone in patients with heart disease has been proposed as an intermediate outcome linking heart rate variability with increased mortality. ${ }^{24}$ Resting heart rate also has been linked to CVD in patients with SDB and chornic obstructive pulmonary disease (COPD). ${ }^{21} 2526$ Second, the augmented shear forces due to intermittent episodes of tachycardia secondary to respiratory events and the resultant mechanical shear forces may lead to

Table 6 The adjusted time to event Cox Proportional Hazards Models for RRDI during REM sleep predicting the incidence of CVD event

\begin{tabular}{|c|c|c|c|c|}
\hline & \multirow{2}{*}{$\begin{array}{l}\text { CVD } \\
\text { events }\end{array}$} & \multicolumn{3}{|l|}{$\begin{array}{l}\text { HR }(95 \% \mathrm{Cl}) \\
\text { p value }\end{array}$} \\
\hline & & Unadjusted model (1) & Adjusted model (2) & Adjusted model (3) \\
\hline $\begin{array}{l}\text { Continuous RRDI (10-unit } \\
\text { increment) }\end{array}$ & $\begin{array}{l}24 / 569 \\
(4)\end{array}$ & $\begin{array}{l}1.19(1.07 \text { to } 1.32) \\
0.0013\end{array}$ & $\begin{array}{l}1.17(1.05 \text { to } 1.31) \\
0.0056\end{array}$ & $\begin{array}{l}1.19(1.07 \text { to } 1.33) \\
0.0016\end{array}$ \\
\hline \multicolumn{5}{|l|}{ RRDI category } \\
\hline $\begin{array}{l}\text { Tertile } 1 \\
(<9.0)\end{array}$ & $\begin{array}{l}5 / 187 \\
(3)\end{array}$ & REF & REF & REF \\
\hline $\begin{array}{l}\text { Tertile 2 } \\
(9.0-<24.0)\end{array}$ & $\begin{array}{l}7 / 194 \\
(4)\end{array}$ & $\begin{array}{l}1.34(0.42 \text { to } 4.24) \\
0.6222\end{array}$ & $\begin{array}{l}1.19(0.37 \text { to } 3.78) \\
0.7732\end{array}$ & $\begin{array}{l}1.24 \text { (0.39 to } 4.00) \\
0.7171\end{array}$ \\
\hline $\begin{array}{l}\text { Tertile } 3 \\
(\geq 24.0)\end{array}$ & $\begin{array}{l}12 / 188 \\
(6)\end{array}$ & $\begin{array}{l}2.92(1.00 \text { to } 8.55) \\
0.0508\end{array}$ & $\begin{array}{l}2.42(0.80 \text { to } 7.29) \\
0.1173\end{array}$ & $\begin{array}{l}2.69(0.88 \text { to } 8.19) \\
0.0825\end{array}$ \\
\hline P-trend & & 0.0393 & 0.0936 & 0.0657 \\
\hline
\end{tabular}

Model (1) is unadjusted. Model (2) is adjusted for age, sex and body mass index. Model (3) is additionally adjusted for age, sex, body mass index and $\mathrm{AHI}(4 \%$ criteria).

$\mathrm{AHI} 4 \%$, apnoea-hypopnoea index with hypopnoea scored if associated with at least 4\% desaturation (events/hour); CVD, cardiovascular events; REM, rapid eye movement; RRDI, R-R interval dips index (dips/hour). 
Table $7 \quad$ A summary of CVD events types

CVD events types $(n=24)$

\begin{tabular}{ll}
\cline { 2 - 2 } First event type* $^{*} \mathbf{n}(\%)$ \\
\hline Myocardial infarction & $9(36)$ \\
Heart failure & $4(17)$ \\
\hline Pacemaker & $2(8)$ \\
CAD/intervention & $12(48)$ \\
Bypass surgery & $1(4)$ \\
CVD death & $5(20)$ \\
\hline
\end{tabular}

*Individuals could have multiple type events (for example myocardial infarction and stent and coronary artery disease). If multiple events were reported over the course of follow-up, the first reported event was used in this analysis.

endothelial dysfunction. ${ }^{27}$ This possibility is physiologically plausible particularly in male patients, ${ }^{28}$ who may have significant endothelial dysfunction secondary to activation of several inflammatory pathways. Such pathophysiologic changes in untreated SDB patients have been linked to nocturnal angina, myocyte necrosis leading to cardiomyopathy and cardiac remodelling. ${ }^{29-31}$ Our findings corroborate these pathological changes particularly in the coronary vessels making the vast majority of CVD events either related to coronary artery disease (CAD) $(48 \%)$ or myocardial infarction $(36 \%)$, predominantly in men. On the other hand, medications such as Betablockers attenuate the increase in heart rate related to respiratory events during sleep in patients with hypertension and untreated SDB. ${ }^{32}$ This modulation of cardiac responses in patients with SDB provides a mechanism by which Beta-blockers may decrease the risk of sudden cardiac death, particularly in patients with CVD.${ }^{33}$ Finally, hypoxic events can affect the autonomic cardiac response and generate significant RRI dips events. ${ }^{34}$ Hypoxemia and RRI dips may represent different features of SDB-related stress, both of which may contribute to CVD morbidity and mortality through independent pathways. Our findings suggest a need to further identify the intermediate mechanisms that link RRI dips events to longterm outcomes.

This study has several strengths including its prospective design with longitudinal follow-up of participants, community-based including a diverse group of ages and morbidities from both genders, and the use of the gold-standard laboratory-based polysomnography for assessment of SDB. This study assessed the role of heart rate changes, a heritable and physiological phenotype, on CVD outcome. These findings can allow clinicians

Table 8 Adjusted time to event Cox Proportional Hazards Models for RRDI Predicting Incidence of CVD Event Stratified by Gender for Continuous RRDI and across Categories of Participants with RRDI more than 20 dips/hour.

\begin{tabular}{|c|c|c|c|}
\hline & Males $(n=300)$ & Females $(n=269)$ & $P$ value \\
\hline Age, mean (SD) & $58(8)$ & $58(8)$ & 0.52 \\
\hline Body mass index, $\mathrm{kg} / \mathrm{m}^{2}$, mean (SD) & $30(5)$ & $32(7)$ & 0.0001 \\
\hline AHI, mean (SD) & $13(16)$ & $10(12)$ & 0.0045 \\
\hline RRDI (SLEEP), mean (SD) & $26(24)$ & $18(21)$ & $<0.0001$ \\
\hline RRDI (ALL), mean (SD) & $30(23)$ & $22(20)$ & $<0.0001$ \\
\hline RRDI (SLEEP)>20, n (\%) & $143(48)$ & $78(29)$ & $<0.0001$ \\
\hline \multirow[t]{2}{*}{ RRDI (ALL)>20, n (\%) } & $179(60)$ & $105(39)$ & $<0.0001$ \\
\hline & $\begin{array}{l}\text { Adjusted model }(3)^{\star}(95 \% \mathrm{Cl}) \\
\text { p value }\end{array}$ & $\begin{array}{l}\text { Adjusted model }(3) *(95 \% \mathrm{Cl}) \\
\text { p value }\end{array}$ & \\
\hline Continuous RRDI (SLEEP) (10-unit increment) & $\begin{array}{l}1.19(1.04 \text { to } 1.36) \\
0.011\end{array}$ & $\begin{array}{l}1.22(0.96 \text { to } 1.54) \\
0.109\end{array}$ & \\
\hline \multicolumn{4}{|l|}{ RRDI category } \\
\hline$<20$ & REF & REF & \\
\hline$>20$ & $\begin{array}{l}1.85(0.67 \text { to } 5.07) \\
0.234\end{array}$ & $\begin{array}{l}1.29(0.22 \text { to } 7.47) \\
0.779\end{array}$ & \\
\hline Continuous RRDI (ALL) (10-unit increment) & $\begin{array}{l}1.22(1.06 \text { to } 1.40) \\
0.006\end{array}$ & $\begin{array}{l}1.25(0.97 \text { to } 1.67) \\
0.086\end{array}$ & \\
\hline \multicolumn{4}{|l|}{ RRDI category } \\
\hline$<20$ & REF & REF & \\
\hline$>20$ & $\begin{array}{l}4.34(1.32 \text { to } 14.34) \\
0.016\end{array}$ & $\begin{array}{l}2.03(0.38 \text { to } 10.77) \\
0.407\end{array}$ & \\
\hline
\end{tabular}

*Model adjusted for age, body mass index and $\mathrm{AHI} 4 \%$ categories.

$\mathrm{AHI} 4 \%$, apnoea-hypopnoea index with hypopnoea scored if associated with at least $4 \%$ desaturation (events/hour); RRDI, R-R interval dips index (dips/hour). 
to identify early on high-risk patients and implement an intervention to prevent cardiovascular disease and premature death. In addition, our study used a novel method of automatic detection of heart rate accelerations that can be translated into an executable program or a plug-in for sleep scoring software and can be used in any sleep study across the world. The study has some limitations. First, we used the self-reported diagnosis of CVD (including dates of diagnosis). However, there is evidence that self-reported CVD is very reliable and accurate as noted in the AusDiab cohort. Barr et al reported more than $99 \%$ of self-reported CVD events were correctly verified in the patients' medical records; only $0.2 \%$ of those denying any CVD event being recorded as having had an event on the medical record. ${ }^{35}$ Second, we lack racial diversity in our study as $95 \%$ was reported as a white race. Therefore, the results may not be generalisable to other races. Third, the incidence of CVD in this population is relatively smaller than what was observed in other high cardiovascular risk population. This is might be due to the inclusion of only those who have no prior history of CVD. Finally, the study excluded participants who had pre-existing cardiac disease history (history of any CVD event as listed in table 7), were on CPAP treatment or were on beta-blocker and/or other chronotropic medications, which alter the cardiac autonomic responses, particularly heart rate bursts following respiratory events. Therefore, this study could not include all WSCS participants and might not be applicable to individuals with heart disease or if taking Beta-blocker or chronotropic medications. Likewise, this study might not be applicable to individuals with arrhythmia, frequent ectopic beats and in case of cardiac pacemakers.

\section{Clinical perspectives}

The association between heart rate changes and the cardiovascular outcome may have significant clinical implications. First, the association between increased incidence of cardiovascular events and the RRDI suggest that early detection of heart rate fluctuations during sleep could identify those who are at increased risk of future CVD events and inform primary preventions strategies. Second, several behavioural factors ${ }^{36}$ and medical conditions, such as $\mathrm{SDB}^{37}$ and $\mathrm{COPD},{ }^{26}$ are associated with changes in resting heart rate, which increase the risk of cardiovascular diseases. Third, the attenuation of heart rate accelerations by Beta-blockers during sleep as recently shown in patients with $\mathrm{SDB},{ }^{32}$ indicate that Betablockers may play an important role in preventing CVD. However, large prospective clinical studies are needed to confirm this finding.

In summary, this study demonstrates that after adjusting for age, BMI, sex, AHI and other comorbidities, people with high RRDI during sleep study are at increased risk for incident CVD events. These results suggest that assessing the ECG of high-risk patients for RRDI during sleep may assist in predicting cardiovascular disease early on. Further research is needed to understand the pathophysiology of heart rate bursts during sleep and whether the RRI dips provide markers of subclinical cardiac disease or whether their occurrence represents pathophysiological responses to respiratory events that increase the risk of cardiovascular morbidity.

Acknowledgements The authors would like to thank Amanda Rasmuson for her assistance throughout the study. The authors would like also to thank Dr. Aiden Abidov and Naresh Punjabi for their valuable advice.

Contributors Conception and design: AS, MSB, and PP. The acquisition, analysis, or interpretation of data: AS, MSB, LAR, SM, NA, BA, SF, MH, GA, PP. Statistical analysis: LAR, EH, and PP. Obtained funding: AS and PP. Study supervision: AS and PP.

Funding Research reported in this publication was supported by the National Heart, Lung, and Blood Institute of Health under Award Number R21HL140447. The Wisconsin Sleep Cohort Study was supported by the National Heart, Lung, and Blood Institute (R01HL62252), National Institute on Aging (R01AG036838) and the National Center for Research Resources (UL1RR025011) at the US NIH. Author (AS) is supported by Career Development Award \# IK2CX000547 from the Clinical Science Research \& Development Service of the VA Office of Research and Development from the (US) Department of Veterans Affairs and by Cardiovascular Research Institute (CVRI).

Disclaimer The following authors AS, SB, SM and Wayne State University have a pending Patent \#US62395634, entitled 'The Detection of Sleep Disordered Breathing Using Cardiac Autonomic Responses', application number \#15/706097 for Utility/Design using an application data sheet (37 CFR 1.54), Date Filed: September 15, 2017.

Competing interests None declared.

Patient consent for publication Not required.

Provenance and peer review Not commissioned; externally peer reviewed.

Data sharing statement Data may be obtained from a third party and are not publicly available.

Open access This is an open access article distributed in accordance with the Creative Commons Attribution Non Commercial (CC BY-NC 4.0) license, which permits others to distribute, remix, adapt, build upon this work non-commercially, and license their derivative works on different terms, provided the original work is properly cited, appropriate credit is given, any changes made indicated, and the use is non-commercial. See: http://creativecommons.org/licenses/by-nc/4.0/.

\section{REFERENCES}

1. Somers VK, White DP, Amin R, et al. Sleep apnea and cardiovascular disease: an American Heart Association/american College Of Cardiology Foundation Scientific Statement from the American Heart Association Council for High Blood Pressure Research Professional Education Committee, Council on Clinical Cardiology, Stroke Council, and Council On Cardiovascular Nursing. In collaboration with the National Heart, Lung, and Blood Institute National Center on Sleep Disorders Research (National Institutes of Health). Circulation 2008;118:1080-111.

2. Sankari A, Badr MS. Diagnosis of sleep disordered breathing in patients with chronic spinal cord injury. Arch Phys Med Rehabil 2016;97:176-7.

3. Mazzotti DR, Keenan BT, Lim DC, et al. Symptom subtypes of obstructive sleep apnea predict incidence of cardiovascular outcomes. Am J Respir Crit Care Med 2019.

4. Won CHJ, Qin L, Selim B, et al. Varying hypopnea definitions affect obstructive sleep apnea severity classification and association with cardiovascular disease. J Clin Sleep Med 2018;14:1987-94.

5. Mansukhani MP, Kolla BP, Wang Z, et al. Effect of varying definitions of hypopnea on the diagnosis and clinical outcomes of sleepdisordered breathing: a systematic review and meta-analysis. J Clin Sleep Med 2019;15:687-96.

6. Butler MP, Emch JT, Rueschman M, et al. Apnea-hypopnea event duration predicts mortality in men and women in the sleep heart health study. Am J Respir Crit Care Med 2019;199:903-12.

7. Singh JP, Larson MG, O'Donnell CJ, et al. Heritability of heart rate variability: the Framingham Heart Study. Circulation 1999;99:2251-4. 
8. Fava C, Burri P, Almgren P, et al. Dipping and variability of blood pressure and heart rate at night are heritable traits. Am J Hypertens 2005;18:1402-7.

9. Zinchuk AV, Jeon S, Koo BB, et al. Polysomnographic phenotypes and their cardiovascular implications in obstructive sleep apnoea. Thorax 2018;73:472-80.

10. Sankari A, Finn L, Maresh S, et al. A New Marker Of Cardiovascular Disease In Patients With Sleep-Disordered Breathing: Results From The Wisconsin Sleep Cohort Study. Am J Respir Crit Care Med 2016;193:A2523.

11. Sankari A, Maresh S, Finn L, et al. Association of nocturnal RR intervals changes and cardiovascular outcome in a large prospective community-based cohort. Am J Respir Crit Care Med 2019;199:A7273.

12. Young T, Palta M, Dempsey J, et al. The occurrence of sleepdisordered breathing among middle-aged adults. N Engl J Med 1993;328:1230-5.

13. Sankari A, Badr MS, Maresh S. Detection of sleep disordered breathing using cardiac autonomic responses. In: US Patent App. 15/706,097, 2018.

14. Sankari A, Pranathiageswaran S, Maresh S, et al. Characteristics and Consequences of Non-apneic Respiratory Events During Sleep. Sleep 2017; 40 .

15. Flemons W, Buysse D, Redline S, et al. Sleep-related breathing disorders in adults. Sleep 1999;22:667-89.

16. Cox PR. Life tables: Wiley Online Library, 1972.

17. Korn EL, Graubard BI, Midthune D. Time-to-event analysis of longitudinal follow-up of a survey: choice of the time-scale. Am J Epidemiol 1997:145:72-80.

18. Kaplan EL, Meier P. Nonparametric estimation from incomplete observations. J Am Stat Assoc 1958;53:457-81.

19. Punjabi NM, Newman AB, Young TB, et al. Sleep-disordered breathing and cardiovascular disease: an outcome-based definition of hypopneas. Am J Respir Crit Care Med 2008;177:1150-5.

20. Azarbarzin A, Sands SA, Stone KL, et al. The hypoxic burden of sleep apnoea predicts cardiovascular disease-related mortality: the Osteoporotic Fractures in Men Study and the Sleep Heart Health Study. European heart journal 2018

21. Narkiewicz K, Montano N, Cogliati C, et al. Altered cardiovascular variability in obstructive sleep apnea. Circulation 1998;98:1071-7.

22. Tamisier R, Weiss JW, Pépin JL. Sleep biology updates: hemodynamic and autonomic control in sleep disorders. Metabolism 2018;84:3-10.
23. Morgan BJ, Crabtree DC, Puleo DS, et al. Neurocirculatory consequences of abrupt change in sleep state in humans. J Appl Physiol 1996;80:1627-36.

24. Tsuji $\mathrm{H}$, Larson MG, Venditti FJ, et al. Impact of reduced heart rate variability on risk for cardiac events. The Framingham Heart Study. Circulation 1996;94:2850-5.

25. Wolf J, Lewicka J, Narkiewicz K. Obstructive sleep apnea: an update on mechanisms and cardiovascular consequences. Nutr Metab Cardiovasc Dis 2007;17:233-40.

26. Jensen MT, Marott JL, Lange $\mathrm{P}$, et al. Resting heart rate is a predictor of mortality in COPD. Eur Respir J 2013;42:341-9.

27. Fisher $A B$, Chien $S$, Barakat Al, et al. Endothelial cellular response to altered shear stress. Am J Physiol Lung Cell Mol Physiol 2001;281:L5 29-L533.

28. Alkatib S, Sankri-Tarbichi AG, Badr MS. The impact of obesity on cardiac dysfunction in patients with sleep-disordered breathing. Sleep Breath 2014;18:137-42.

29. Dincer HE, O'Neill W. Deleterious effects of sleep-disordered breathing on the heart and vascular system. Respiration 2006;73:124-30.

30. Franklin KA, Nilsson JB, Sahlin C, et al. Sleep apnoea and nocturnal angina. Lancet 1995;345:1085-7.

31. Kuniyoshi FH, Garcia-Touchard A, Gami AS, et al. Day-night variation of acute myocardial infarction in obstructive sleep apnea. J Am Coll Cardiol 2008;52:343-6.

32. Wolf J, Drozdowski J, Czechowicz K, et al. Effect of beta-blocker therapy on heart rate response in patients with hypertension and newly diagnosed untreated obstructive sleep apnea syndrome. Int $J$ Cardiol 2016;202:67-72

33. Gottlieb SS, McCarter RJ, Vogel RA. Effect of beta-blockade on mortality among high-risk and low-risk patients after myocardial infarction. N Engl J Med 1998;339:489-97.

34. Sankari A, Bascom AT, Riehani A, et al. Tetraplegia is associated with enhanced peripheral chemoreflex sensitivity and ventilatory longterm facilitation. J Appl Physiol 2015;119:1183-93.

35. Barr EL, Tonkin AM, Welborn TA, et al. Validity of self-reported cardiovascular disease events in comparison to medical record adjudication and a statewide hospital morbidity database: the AusDiab study. Intern Med J 2009;39:49-53.

36. Ohira T, Diez Roux AV, Prineas RJ, et al. Associations of psychosocial factors with heart rate and its short-term variability: multi-ethnic study of atherosclerosis. Psychosom Med 2008;70:141-6.

37. Kawano Y, Tamura A, Watanabe T, et al. Influence of the severity of obstructive sleep apnea on heart rate. J Cardiol 2010;56:27-34. 